Methods All incident cases of thyroid cancer (C73) in the Arkhangelsk region in 2000-2009 as well as information about deaths were extracted from the regional cancer registry. Population size was obtained from the Regional Bureau of Statistics. Incidence and mortality were calculated per 100 000. Survival was analysed using Kaplan-Meier curves with log rank tests.

Results Altogether, 529 new cases of thyroid cancer occurred in the region during the study period. Females comprised $82.8 \%$ of cases. Papillary carcinoma, follicular carcinoma and other forms was found in $56.3 \%, 27.7 \%$ and $21.9 \%$ of patients, respectively. Stages $0,1,2,3$, and 4 were diagnosed in $1.1 \%, 58.0 \%, 16.4 \%, 13.2 \%$, and $11.0 \%$ of patients respectively. The incidence increased from 4.3 in 2000 to 4.7 in 2009. Mortality decreased from 0.42 in 2000 to 0.24 in 2009. By 2010, 50 (9.5\%) died from thyroid cancer and 33 (6.2\%) were censored. Mean overall survival was 108 (95\% CI 105 to 111) months. Males, patients aged $45+$ years and those having stages 3-4 had significantly lower survival than the reference groups (all at $\mathrm{p}<0.001$ ). Average survival time for patients with papillary, follicular and other morphology types was 117, 107 and 83 months, respectively $(p<0.001)$

Conclusions Incidence of thyroid cancer in Arkhangelsk region of Russia slightly increased while the mortality decreased. Age, stage, morphology and gender were all associated with survival.

\section{P2-108 TRENDS IN MORTALITY FROM BREAST AND CERVICAL CANCER IN THE BRAZILIAN CAPITAL CITIES, 1996-2007}

doi:10.1136/jech.2011.142976i.43

${ }^{1} \mathrm{R}$ Guimarães, ${ }^{*}{ }^{2} \mathrm{C}$ Muzi, ${ }^{3} \mathrm{~A}$ Ayres, ${ }^{4} \mathrm{M}$ Ribeiro, ${ }^{4} \mathrm{C}$ Chagas, ${ }^{4} \mathrm{~J}$ Capilupi, ${ }^{4} \mathrm{P}$ Salles, ${ }^{4} \mathrm{Z}$ Melo, ${ }^{4} \mathrm{M}$ Matos. ${ }^{1}$ Federal University of Rio de Janeiro, Institute of Studies in Public Health, Rio de Janeiro, Brazil; ${ }^{2}$ National Cancer Institute, Rio de Janeiro, Brazil; ${ }^{3}$ Gaffrée Guinle University Hospital, Rio de Janeiro, Brazil;, ${ }^{4}$ Federal University of Rio de Janeiro, Rio de Janeiro, Brazil

Objective To analyse the trend in mortality from breast cancer and cervical cancer in Brazilian capitals from 1996 to 2007.

Materials and Methods We calculated mortality rates for breast cancer and cervical cancer in Brazilian capital cities from 1996 to 2007, standardised for the Brazilian population and for the world's population. Trend analysis was performed using the polynomial regression model.

Results Cancer of the cervix showed a considerable reduction in mortality rate in most capital cities, although in some cities the rate rose. Breast cancer mortality remained high in most of the capital cities. Trends were predominantly linear.

Conclusion It may be that mortality from cancer of the cervix may be failing due to increased adherence with Pap smear screening. The high breast cancer mortality rates may relate to its associations with habits, lifestyles and culture.

\section{P2-109 INCREASE INCOME AND MORTALITY OF COLORECTAL CANCER IN BRAZIL}

doi:10.1136/jech.2011.142976i.44

${ }^{1} \mathrm{R}$ Guimarães, ${ }^{*} \mathrm{C}$ Muzi, ${ }^{2} \mathrm{R}$ Ramos, ${ }^{3} \mathrm{~S}$ Annes, ${ }^{4} \mathrm{C}$ Queiróz. ${ }^{1}$ Federal University of Rio de Janeiro, Institute of Studies in Public Health, Rio de Janeiro, Brazil; ${ }^{2}$ National Cancer Institute, Rio de Janeiro, Brazil; ${ }^{3}$ Rio de Janeiro State Health Department, Rio de Janeiro, Brazil; ${ }^{4}$ Federal University of Rio de Janeiro, Rio de Janeiro, Brazil

Objective The objective of this study is to determine the correlation between average per capita income and the rate of colorectal cancer mortality in Brazil between 2000 and 2007.

Materials and Methods We obtained data on median household income and mortality rate for colon, rectum and anus between 2001 and 2007 by DATASUS. A trend analysis was performed using linear regression, and correlation between variables by Pearson's correlation coefficient.

Results There was a tendency towards an increase average family income and standardised mortality rate of colon, rectum and anus in Brazil. There was also strong positive correlation $(r=0.81, p<0.001)$ between income and mortality for this cancer located throughout the study period.

Conclusion The increase in income may partially explain the increased occurrence of cancer of the colon, rectum and anus, and this is possibly due to differential access to food recognised as a risk factor, such as red meat and fat food. É therefore important Assess the priority of public health programs addressing nutrition in countries with intermediate economy, as is the case in Brazil.

\section{P2-110 NUMBER OF TEETH IS ASSOCIATED WITH THE LEVEL OF SALIVARY NITRIC OXIDE}

doi:10.1136/jech.2011.142976i.45

D-H Han, ${ }^{*}$ H-D Kim, H-M Kim, H-S Shin, M-S Kim. Seoul National University, Seoul, Republic of Korea

Introduction Nitric oxide (NO) is involved in second messenger formation, osteoblast and osteoclast function, and blood flow. This raises the question of whether or not altered salivary NO levels interfere with tooth loss by influencing the bone remodelling cycle. This study investigates if different levels of salivary NO are associated with existing number of teeth among Korean elderly.

Methods A total of 178 subjects (68 males and 110 females; age range 48-84 years) received a dental and general examination in 2009 and 2010. Number of remaining teeth and salivary flow rate were evaluated by a dentist. Fasting plasma glucose level and blood pressure were also measured. Age, gender, education level, and smoking were evaluated through interview. Salivary NO was determined by the Griess reagent. ANCOVA and Multiple linear regression analyses were applied.

Results After controlling for age, gender, education level, smoking, physical activity, salivary flow rate, fasting plasma glucose level, systolic blood pressure and diastolic blood pressure, higher NO level group showed increased number of teeth compared to moderate and lower NO level group, which is not significant. However, salivary NO levels had a dose-response relationship with the existing number of teeth in Korean elderly $(\beta=-0.007, p=0.046)$.

Conclusion This study presents for the first time a dose-dependent relationship between salivary NO levels and number of teeth, indicating a link between oral health and salivary $\mathrm{NO}$ levels, and that the levels of salivary $\mathrm{NO}$ could be a proper indicator for oral health in this respect.

\section{P2-111 IMPROVING THE HEALTH OF JAPANESE WOMEN: ACCEPTANCE OF HPV VACCINATION IN MOTHERS OF ADOLESCENT GIRLS}

doi:10.1136/jech.2011.142976i.46

${ }^{1,2} \mathrm{~S}$ Hanley, ${ }^{*}{ }^{1} \mathrm{E}$ Yoshioka, ${ }^{2} \mathrm{Y}$ Ito, ${ }^{3} \mathrm{R}$ Konno, ${ }^{4} \mathrm{~N}$ Sakuragi, ${ }^{5} \mathrm{R}$ Kishi. ${ }^{1}$ Hokkaido University, Graduate School of Medicine, Department of Public Health, Sapporo, Japan; ${ }^{2}$ Japanese Red Cross Hokkaido College of Nursing, Faculty of Nursing, Kitami, Japan; ${ }^{3}$ Jichi Medical University, Saitama Medical Center, Department of Obstetrics and Gynecology, Saitama, Japan; ${ }^{4}$ Hokkaido University, Graduate School of Medicine, Department of Reproductive Endocrinology and Oncology, Sapporo, Japan; ${ }^{5}$ Hokkaido University, Center for Environmental and Health Sciences, Sapporo, Japan

Introduction Cervical Cancer (CC) caused by oncogenic Human Papillomavirus (HPV) is the most common cancer in young Japanese 